\title{
Influence of Asymmetric Distribution of Defects on Dynamic Stability of Bernoulli-Euler Beam
}

\author{
W. Sochacki, J. Garus and S. Garus* \\ Department of Mechanics and Fundamentals of Machinery Design, \\ Faculty of Mechanical Engineering and Computer Science, \\ Częstochowa University of Technology, J.H. Dabrowskiego 73, 42-201 Częstochowa, Poland \\ Doi: 10.12693/APhysPolA.139.557 \\ *e-mail: $\quad$ s.garus@imipkm.pcz.pl
}

\begin{abstract}
Defects of structural elements may have a significant impact on the dynamic stability of the systems in which these elements are used. A Bernoulli-Euler beam was taken as an example of a structural element with defects. The study analyzed the influence of the asymmetric distribution of defects modeled with rotating springs. The influence of the depth of damage on the dynamic stability of the beam was also investigated. The method of mode summation was used to solve the problem of dynamic stability. After using the orthogonality condition of the eigenfunctions, the equations of motion of the studied system were presented in the form of the Mathieu equations. The obtained coefficients $a$ and $b$ of the Mathieu equations allow to determine stable and unstable solutions to the equation on the Strutt card. On this basis, it is possible to determine the dynamic stability of the tested beam for specific physical and geometric parameters of the system.
\end{abstract}

topics: cracked beam, dynamic stability, Mathieu equation

\section{Introduction}

A crack initiation and propagation in the material is most often the result of variable loads. Cracks reduce the stiffness of components and are the most common cause of structural defects. This type of damage to a structural element is a serious threat to the structure or proper functioning of the machine. Very often, these structural elements are beams.

During the last few decades, a great deal of research work was carried out on beams and beam structures with cracks. The use of the vibration technique to detect the location and size of a fatigue crack in structures is presented in [1]. Both the depth and location of cracks are compared with data obtained from FEM models. The effect of a transverse open crack on free vibrations of a simply supported Bernoulli-Euler beam is presented in [2]. An influence of the parameters of the loading heads on the stability and free vibrations as well as on loading capacity of a cracked column subjected to a specific load is studied in [3]. Furthermore, in [4], flexural vibrations measurements of a cantilever beam with a rectangular cross-section that has a transverse surface crack extending uniformly along the width of the beam are conducted. Analytical results were used to relate the measured vibration modes to the crack location and depth. In [5], the description of a detection method of a crack presence on the surface of a beam-type structural element using natural frequency is presented. The vibration tests of a beam structure with damping as an $L$-type frame with cracks are presented in [6]. In [7], in turn, the analytical transfer matrix method is performed to solve direct and inverse problems of simply supported beams with an open crack.

A crack in a structural element, which is a beam, affects the vibrations of the structure, and thus its dynamic stability. There are many studies in the literature on the dynamic stability of beams. In [8], the dynamic stability of an elastic beam was analyzed. The analysis of the dynamic stability of beams with step changes in the cross-section under moving loads was carried out in [9]. The stability problem associated with a Bernoulli-Euler beam made of an arbitrary linear viscoelastic material was formulated in [10]. The three-parameter and the Kelvin-Voigt models have been analyzed in the presence of constant and periodic loads. In [11], the effect of a crack on the dynamic stability of a freefree Timoshenko beam - when it is subjected to a constant or pulsating follower force - was investigated. Further, in [12], the parametric instability of the electromagnetically excited beam was analyzed. In $[13,14]$, the problem of a simply supported beam and a cantilever beam loaded axially with harmonic force - showing the destabilizing effect of additional elements attached to the beams was analyzed. Analytical and experimental studies 
on the influence of the mass installed at the end of a beam on its dynamic stability were carried out in [15]. Moreover, in [16], the dynamic stability of a beam supported elastically at its ends was analyzed. The parametric vibrations of a beam loaded with a concentrated mass, showing the influence of the beam weight and inertia of the rotating mass on the beam vibrations were investigated in [17].

In this paper, a simply supported beam with cracks, loaded with a longitudinal force in the form $P(t)=P_{0}+S \cos (\nu t)$, is considered. Cracks modeled with rotary springs are located on the beam anywhere between the supports. The tested beam was treated as a Bernoulli-Euler beam. The problem of dynamic stability was solved by the mode summation method. The applied research procedure allowed to describe the dynamics of the tested system using the Mathieu equation. The influence of a crack depth and its location on the beam on the value of the $b$ coefficient in the Mathieu equation for the constant value of the coefficient is investigated.

\section{Mathematical model of beam vibration}

Figure 1 shows the analyzed beam with cracks, the properties of which are modeled by springs.

For the $i$ th part of the beam, the vibration equation takes the form

$$
\begin{aligned}
& E_{i} J_{i} \frac{\partial^{4} w_{i n}\left(x_{i}, t\right)}{\partial x_{i}^{4}}+P(t) \frac{\partial^{2} w_{i n}\left(x_{i}, t\right)}{\partial x_{i}^{2}} \\
& \quad+\rho_{i} A_{i} \frac{\partial^{2} w_{i n}\left(x_{i}, t\right)}{\partial t^{2}}=0
\end{aligned}
$$

where $A_{i}$ is the cross-section area, $\rho_{i}$ is the density, $i=1,2,3$ is the $i$ th part of the beam. The explicit form of $P(t)=P_{0}+S \cos (\nu t)$ involves the forcing frequency $\nu$.

Position and time variables were separated using the equation

$$
w_{i n}\left(x_{i}, t\right)=W_{i n}\left(x_{i}\right) \cos \left(\omega_{n} t\right),
$$

where $\omega_{n}$ is the beam's $n$th natural frequency.

By inserting (2) into (1) we obtain the timeindependent equation of motion

$$
\begin{gathered}
E_{i} J_{i} W_{i n}^{I V}\left(x_{i}\right)+P_{0} W_{i n}^{I I}\left(x_{i}\right) \\
-\rho_{i} A_{i} \omega_{n}^{2} W_{i n}\left(x_{i}\right)=0,
\end{gathered}
$$

on the basis of which the beam's natural frequencies were determined. Also, it applies that

$$
\begin{aligned}
& W_{1}(0)=0, \quad W_{1}^{I I}(0)=0, \quad W_{i}\left(l_{i}\right)=W_{i+1}(0), \\
& C_{i i+1}\left(W_{i}^{I}\left(l_{i}\right)-W_{i+1}^{I}(0)\right)+E_{i} J_{i} W_{i}^{I I}\left(l_{i}\right)=0 \\
& E_{i} J_{i} W_{i}^{I I}\left(l_{i}\right)-E_{i+1} J_{i+1} W_{i+1}^{I I}(0)=0 \\
& E_{i} J_{i} W_{i}^{I I I}\left(l_{i}\right)+P_{0} W_{i}^{I}\left(l_{i}\right)-E_{i+1} J_{i+1} W_{i+1}^{I I I}(0) \\
& \quad-P_{0} W_{i+1}^{I}(0)=0, \\
& W_{3}\left(l_{3}\right)=0, \quad W_{3}^{I I}\left(l_{3}\right)=0 .
\end{aligned}
$$

The superscript Roman numerals denote the differentiation with respect to $x$, where $i=1,2,3$.

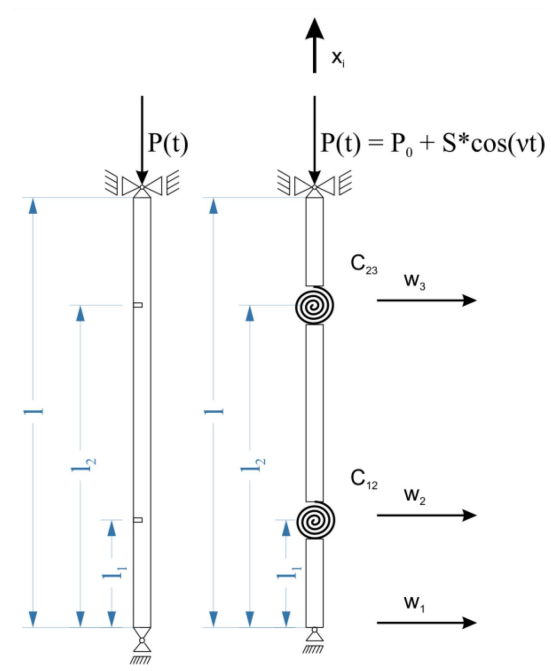

Fig. 1. Model of a beam with cracks modeled by rotational springs.

The solution of (3) can be assumed as

$$
\begin{aligned}
& W_{i n}\left(x_{i}\right)=D_{i 1} \cosh \left(\alpha_{i n} x_{i}\right)+D_{i 2} \sinh \left(\alpha_{i n} x_{i}\right) \\
& +D_{i 3} \cos \left(\beta_{i n} x_{i}\right)+D_{i 4} \sin \left(\beta_{i n} x_{i}\right),
\end{aligned}
$$

where $D_{i k}$ are the integration constants for $k=1, \ldots 4$, and parameters $\alpha$ and $\beta$ are defined as

$$
\begin{aligned}
& \alpha_{i n}^{2}=-\frac{\lambda_{i}}{2}+\sqrt{\frac{\lambda_{i}^{2}}{4}+\Omega_{i n},} \\
& \beta_{i n}^{2}=\frac{\lambda_{i}}{2}+\sqrt{\frac{\lambda_{i}^{2}}{4}+\Omega_{i n}},
\end{aligned}
$$

where $\Omega_{i n}^{2}=\omega_{n}^{2} \rho_{i} A_{i} /\left(E_{i} J_{i}\right), \lambda_{i}=P_{0} /\left(E_{i} J_{i}\right)$.

The boundary problem of the tested beam is formulated on the basis of the vibration equation (3) and boundary conditions (4). Solving it, we obtain the natural frequencies $\omega_{n}$ and the eigenfunctions $W_{i n}\left(x_{i}\right)$ of the beam.

\section{Dynamic stability of the beam}

In order to determine the dynamic stability, the solution of (1) should be adopted in the form of a series of eigenfunctions

$$
w_{i}\left(x_{i}, t\right)=\sum_{n=1}^{\infty} W_{i n}\left(x_{i}\right) T_{n}(t),
$$

which consist of unknown time functions $T_{n}(t)$ and normalized eigenfunctions of free frequencies of $i$ th parts of the beam $W_{i n}\left(x_{i}\right)$. The determined eigenfunctions satisfy the condition

$$
\begin{aligned}
& \sum_{i=1}^{3} \rho_{i} A_{i} \int_{0}^{l_{i}} \mathrm{~d} x_{i} W_{i n}\left(x_{i}\right) W_{i m}\left(x_{i}\right)= \\
& \begin{cases}\gamma_{m}^{2} & \text { for } m=n \\
0 & \text { for } m \neq n .\end{cases}
\end{aligned}
$$


By inserting (9) into (1), one obtains

$$
\begin{aligned}
& \sum_{n=1}^{\infty}\left[\rho_{i} A_{i} W_{i n}\left(x_{i}\right) \ddot{T}_{i n}(t)+E_{i} J_{i} W_{i n}^{I V}\left(x_{i}\right) T_{i n}(t)\right. \\
& \left.\quad+\left(P_{0}+S \cos (\nu t)\right) W_{i n}^{I I}\left(x_{i}\right) T_{i n}(t)\right]=0 .
\end{aligned}
$$

Multiplication by $W_{i m}\left(x_{i}\right)$ allows to obtain

$$
\begin{aligned}
& \sum_{n=1}^{\infty}\left[E_{i} J_{i} W_{i n}^{I V}\left(x_{i}\right) W_{i m}\left(x_{i}\right) T_{n}(t)\right. \\
& \quad+P_{0} W_{i n}^{I I}\left(x_{i}\right) W_{i m}\left(x_{i}\right) T_{n}(t) \\
& \quad+S \cos (\nu t) W_{i n}^{I I}\left(x_{i}\right) W_{i m}\left(x_{i}\right) T_{n}(t) \\
& \left.\quad+\rho_{i} A_{i} W_{i n}\left(x_{i}\right) W_{i m}\left(x_{i}\right) \ddot{T}_{n}(t)\right]=0 .
\end{aligned}
$$

Next, when multiplying the equation of motion (3) by $W_{i m}\left(x_{i}\right)$, one gets

$$
\begin{aligned}
& {\left[E_{i} J_{i} W_{i n}^{I V}\left(x_{i}\right)+P_{0} W_{i n}^{I I}\left(x_{i}\right)\right] W_{i m}\left(x_{i}\right)=} \\
& \rho_{i} A_{i} \omega_{n}^{2} W_{i n}\left(x_{i}\right) W_{i m}\left(x_{i}\right)
\end{aligned}
$$

and after transformation (12), one gets the form

$$
\begin{aligned}
& \sum_{n=1}^{\infty}\left[\rho_{i} A_{i} \omega_{n}^{2} W_{i n}\left(x_{i}\right) W_{i m}\left(x_{i}\right) T_{n}(t)\right. \\
& \quad+S \cos (\nu t) W_{i n}^{I I}\left(x_{i}\right) W_{i m}\left(x_{i}\right) T_{n}(t) \\
& \left.\quad+\rho_{i} A_{i} W_{i n}\left(x_{i}\right) W_{i m}\left(x_{i}\right) \ddot{T}_{n}(t)\right]=0 .
\end{aligned}
$$

For the basic parametric resonance, only the first term of the sum in (13) is taken into account for further analysis. After its integration, the following result is obtained

$$
\begin{aligned}
& T_{1}(t)\left(\omega_{1}^{2} \rho_{i} A_{i} \int_{0}^{l} \mathrm{~d} x_{i} W_{i 1}^{2}\left(x_{i}\right)\right. \\
& \left.+S \cos (\nu t) \int_{0}^{l} \mathrm{~d} x_{i} W_{i 1}^{I I}\left(x_{i}\right) W_{i 1}\left(x_{i}\right)\right) \\
& +\ddot{T}_{1}(t) \rho_{i} A_{i} \int_{0}^{l} \mathrm{~d} x_{i} W_{i 1}^{2}\left(x_{i}\right)=0 .
\end{aligned}
$$

The substitution of $t$ for $\tau=\nu t$ transformed (14) into the Mathieu equation of the form

$$
\ddot{T}(\tau)+(a+b \cos (\tau)) T(\tau)=0,
$$

where $a=\omega_{1}^{2} / \nu^{2}, \ddot{T}(\tau)$ denotes the second derivative after $\tau$, and

$$
b=\frac{S}{\nu^{2}} \frac{\sum_{i=1}^{4} \int_{0}^{l} \mathrm{~d} x_{i} W_{i 1}^{I I}\left(x_{i}\right) W_{i 1}\left(x_{i}\right)}{\sum_{i=1}^{4} \rho_{i} A_{i} \int_{0}^{l} \mathrm{~d} x_{i} W_{i 1}^{2}\left(x_{i}\right)} .
$$

On the basis of the derived (16) in the form of the Mathieu equation and its periodic solutions, stable and unstable regions can be determined (Strutt's chart - Fig. 2) [18]. The value of the coefficient $a$

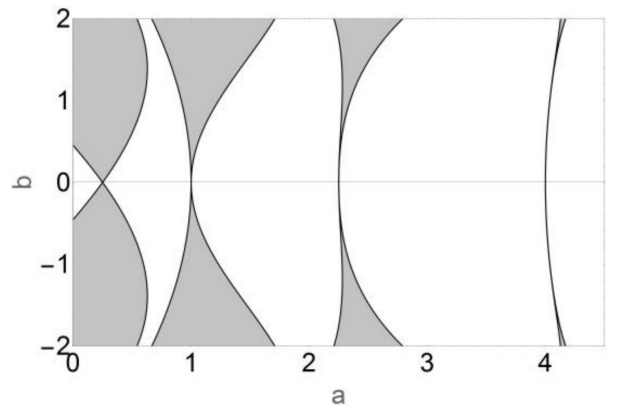

Fig. 2. Strutt's chart showing the stable (white) and unstable (gray) areas for the coefficients $a$ and $b$ of the Mathieu equation.

of the Mathieu equation is influenced by the natural frequency of the beam and the frequency of the exciting force, while the value of the coefficient $b$ is influenced by the type of the material, beam geometry, frequency of the exciting force and the location of defects.

\section{Results and discussion}

The study analyzed a $l=3 \mathrm{~m}$ long beam with two defects located at points $l_{1}$ and $l_{2}$, respectively. The material was steel with density $7.86 \times 10^{3} \mathrm{~kg} / \mathrm{m}^{3}$, Young's modulus was $2.1 \times 10^{11} \mathrm{~Pa}$ and the crosssectional area was $54 \times 10^{-3} \mathrm{~m}^{2}$. The $h$ is the percentage of the crack depth in relation to the beam width. The crack depth $\mathrm{h}$ is defined as the ratio of the cross-sectional area of the notch to the crosssection of the beam. The constant component of the loading force $P$ and its variable component $S$ were set at $5 \%$ of the critical load.

Figure 3 shows the effect of cracks depth and their position on the first and second natural frequencies. As the crack depth increases, the values of both frequency types decrease. The first eigenfrequency reaches the minimum for the location of the cracks at the center of the beam, while the minimum of the second eigenfrequency occurs when the cracks are one-fourth the length of the beam from its ends.

Figure 4 presents the analyzed cases on the Strutt chart. The increase in the frequency of the exciting force, and thus the parameter $a$ of the Mathieu equation, causes a proportional increase in the coefficient $b$. Despite the low value of the exciting force of the columns ( $5 \%$ of the critical force value), the solutions to the equation of motion pass through unstable areas, and it may cause the destruction of the system for selected values of the exciting force frequency.

Figures 5 and 6 show the influence of the crack depth and crack shift on dynamic stability, respectively, on selected examples. Unstable areas are marked in dark color, i.e., those for which the vibration amplitude at a relatively low load increases to infinity due to parametric resonance, which may result in the destruction of the system. 

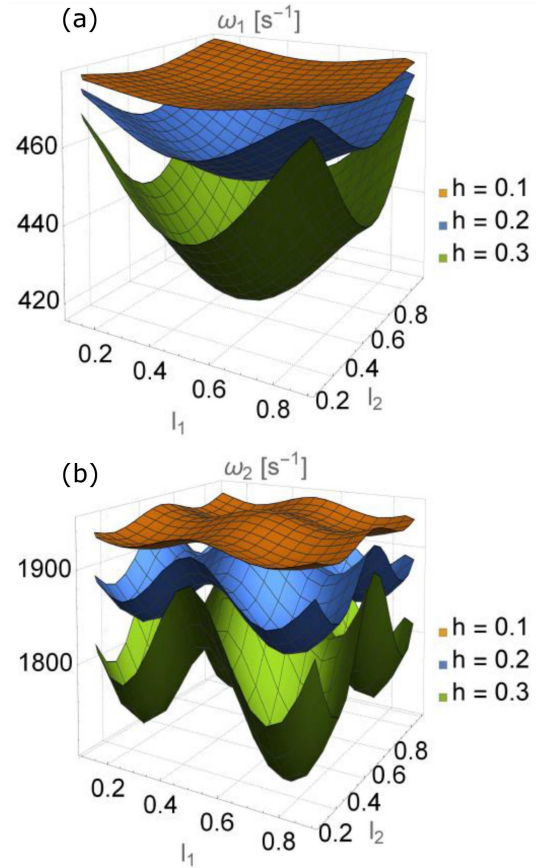

Fig. 3. The first (a) and the second (b) eigenfrequency depending on the location of the cracks and their depth.

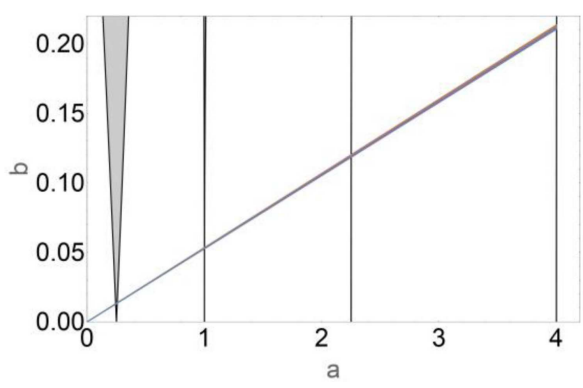

Fig. 4. Analyzed cases plotted on the Strutt chart.

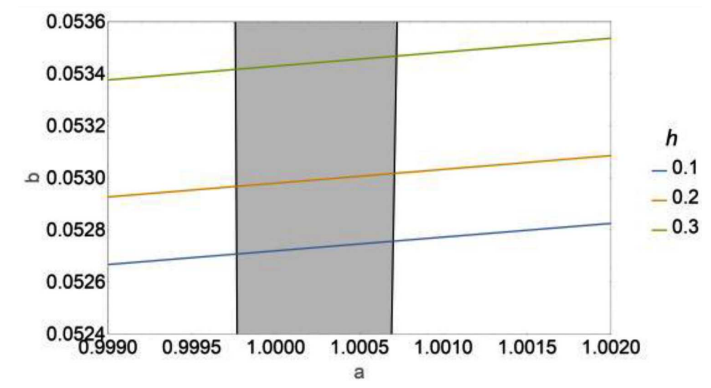

Fig. 5. The effect of crack depth on the dynamic stability of a beam.

In Fig. 5, the crack positions were $l_{1}=1.5 \mathrm{~m}$ and $l_{2}=1.65 \mathrm{~m}$. As shown, increasing the crack depth leads to a non-linear decrease in the dynamic stability of the analyzed beam. At the same time, as shown in Fig. 6, shifting the crack from the center of the beam towards its end increases the dynamic stability.

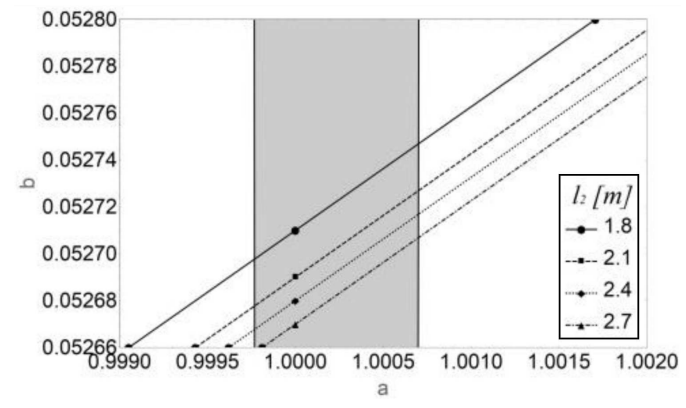

Fig. 6. The influence of the $l_{2}$ crack shift on the dynamic stability of the beam for $l_{1}=0.9 \mathrm{~m}$.

\section{Conclusions}

The paper shows the influence of the crack position on the first and second natural frequencies. The closer the defects are to the center of the beam, the more the value of the first natural frequency decreases. The minima for the second natural frequency are for the distance of defects from the ends of the beam in one-fourth of its length. The reductions of eigenfrequencies are the greater, the greater the depth of defects.

As part of the work, it was shown that there are beam parameters for which the phenomenon of parametric resonance occurs, which may cause its destruction for a relatively low periodic force exciting the system. The dynamic stability increases for smaller defect depths and when they are closer to the beam ends.

\section{Acknowledgments}

The study has been carried out within statutory research of the Department of Mechanics and Machine Design Fundamentals of the Częstochowa University of Technology.

\section{References}

[1] D. Kindova-Petrova, J. Theor. Appl. Mech. 44, 69 (2014).

[2] T.G. Chondros, A.D. Dimarogonas, J. Yao, J. Sound Vib. 215, 17 (1998).

[3] K. Sokół, J. Vibroeng. 20, 1299 (2018).

[4] P.F. Rizos, N. Aspragathos, A.D. Dimarogonas, J. Sound Vib. 138, 381 (1990).

[5] K.H. Barad, D.S. Sharma, V. Vyas, Proc. Eng. 51, 770 (2013).

[6] M. Bold, W. Sochacki, J. Vibroeng. 20, 215 (2018).

[7] H.P. Lin, Eng. Struct. 24, 427 (2004).

[8] G. Cederbaum, M. Mond, J. Appl. Mech. 59, 16 (1992).

[9] U.S. Shirahatil, S.C. Shinha, J. Sound Vib. 174, 57 (1994). 
[10] O.J. Aldraihem, A. Baz, J. Sound Vib. 205, 835 (2002).

[11] K.-H. Kim, J.-H. Kim, J. Sound Vib. 233, 119 (2000).

[12] C.-C. Chen, M.-K. Yeh, J. Sound Vib. 240, 747 (2001).

[13] W. Sochacki, J. Sound Vib. 314, 180 (2008).

[14] W. Sochacki, J. Vibroeng. 15, 280 (2013).
[15] H.A. Evensen, R.M. Evan-Iwanowski, J. Appl. Mech. 33, 141 (1966).

[16] C.E. Majorana, C. Pellegrino, Eng. Computation 14, 792 (1997).

[17] K. Sato, V. Saito, V. Otomi, J. Appl. Mech. 45, 643 (1978).

[18] S.P. Timoshenko, V. Gere, Theory of Elastic Stability, Mc Graw-Hill, 1961. 